\title{
Polarisation-Insensitive Fibre Optic Parametric Amplifiers for Applications in Modern Communication Networks
}

\author{
Vladimir Gordienko ${ }^{1, *}$, Filipe Ferreira ${ }^{1,2}$, Charles Laperle $^{3}$, Maurice O'Sullivan $^{3}$, Kim Roberts ${ }^{3}$, \\ and Nick Doran ${ }^{1}$ \\ ${ }^{I}$ Aston Institute of Photonic Technologies, Aston University, B4 7ET, Birmingham, UK \\ ${ }^{2}$ now with University College London, Gower Street, London, WC1E 6BT, UK \\ ${ }^{3}$ Ciena Corporation, Ottawa, Ontario, K2K 0L1, Canada \\ *e-mail:v.goridenko1@aston.ac.uk
}

\begin{abstract}
We evaluate and compare performance of three polarisation-insensitive fibre optic parametric amplifier (PIFOPA) variants. We employ each variant to amplify a set of $21 \times 50 \mathrm{GHz}$-spaced channels by $12.8 \pm 1.5 \mathrm{~dB}$ and analyse bit-error rate of an amplified 100G PDM-QPSK channel as power per channel is varied. We demonstrate two variants to provide a competitive noise figure of $6 \mathrm{~dB}$. We envisage each of three PI-FOPA variants to be employed for broadband communications, phase-sensitive amplification and extended-reach PON respectively. Keywords: fibre optic parametric amplification, polarisation, noise figure, nonlinear crosstalk.
\end{abstract}

\section{INTRODUCTION}

Fibre optic parametric amplifiers (FOPA) can make a major contribution to future fibre optical communications with a range of features unique to parametric amplifiers. These are: operation in virtually any wavelength range, a theoretically unconstrained gain bandwidth, a capability for phase-sensitive amplification (PSA) and an instantaneous response time [1]. Thus, experiments with FOPA demonstrate possibility of operation at arbitrary wavelengths between $\sim 1300 \mathrm{~nm}$ and $\sim 1700 \mathrm{~nm}[2,3]$, gain bandwidth of $\sim 150 \mathrm{~nm}[4,5]$, noise figure (NF) as low as $1.1 \mathrm{~dB}[6]$ and a transient-free amplification of signal bursts [7, 8].

However, a practical polarisation-insensitive FOPA suitable for multi-span transmission [9] was attained only recently through a novel polarisation-insensitive half-pass loop (HPL) architecture [10, 11]. In HPL-FOPA an arbitrarily polarized signal is split into orthogonal linearly polarized signal components counter-propagating within a loop. Each signal component is then equally amplified by a co-propagating pump. The HPL-FOPA employs two highly nonlinear gain fibres each pumped unidirectionally and thus amplifying only one signal component per gain fibre. Counter-propagating high power pumps are avoided in HPL-FOPA to prevent a nonlinear coupling between them via stimulated Brillouin scattering [12].

Three HPL-FOPA variants have been proposed: "Gain - Loss" (GL) [10, 11], "Loss - Gain" (LG) [13], and "Gain - Filter - Loss" (GFL) [14]. In the GL each signal component is amplified in the first encountered section of HNLF and passes through the second section without amplification to allow for the best noise performance. In the LG a direction of pumps is reversed, so each signal is amplified in the second encountered section of HNLF. This keeps the signal power low for as long as possible and can reduce nonlinear crosstalk accumulated within the loop by $10 \mathrm{~dB}$ as compared to the GL [13]. The GFL is similar to GL, but includes a filter removing idlers generated after the 'gain' section to mitigate nonlinear crosstalk occurring in the second encountered HNLF. The GFL has been shown to reduce nonlinear crosstalk by $4.5 \mathrm{~dB}$ as compared to the GL [14].

This paper compares an optical noise figure of the three HPL-FOPA variants with $12.8 \pm 1.5 \mathrm{~dB}$ net gain whilst amplifying $21 \times 50 \mathrm{GHz}$ spaced WDM channels. Measurements are performed as the total output signal power is varied from $2 \mathrm{dBm}$ to $22 \mathrm{dBm}$. We demonstrate HPL-FOPA noise figure of $\sim 6 \pm 1 \mathrm{~dB}$ in GL and GFL variants and conclude the applicability of each variant. We support our conclusions with BER measurements.

\section{EXPERIMENTAL SETUP}

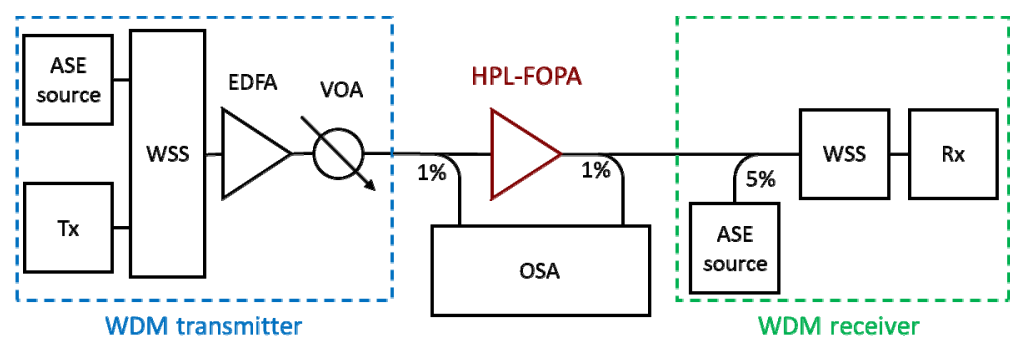

Figure 1. Experimental setup for characterization of HPL-FOPA.

Figure 1 shows an experimental setup for characterization of the HPL-FOPA variants in terms of NF and BER in WDM environment. A WDM transmitter produced a WDM input signal for an examined HPL-FOPA variant, 
and a WDM receiver measured BER of a signal amplified by HPL-FOPA. An optical spectrum analyser (OSA) connected to the input and the output of the HPL-FOPA via calibrated $1 \%$ tap couplers measured optical spectra for calculation of the HPL-FOPA net gain and noise figure.

The WDM transmitter produced 21 x50 GHz-spaced channels between $1533.5 \mathrm{~nm}$ and $1541.3 \mathrm{~nm}$. The middle channel at $1537.4 \mathrm{~nm}$ was a $100 \mathrm{G}$ PDM-QPSK signal sourced from a Ciena transponder WaveLogic 3 . The remaining channels were sourced from amplified spontaneous emission (ASE) noise, shaped using a wavelength selective switch (WSS) and combined with the 100G channel. The WDM channels were amplified by EDFA and attenuated by a variable optical attenuator (VOA) to vary a power per channel between $-25 \mathrm{dBm}$ and $-4 \mathrm{dBm}$.

The WDM receiver added ASE noise via a 5\% coupler to observe both noise-limited and nonlinearity-limited performance across the examined signal power range. Another WSS filtered the $100 \mathrm{G}$ channel and set its power to $-19 \mathrm{dBm}$. Then, the $100 \mathrm{G}$ channel was detected by a coherent receiver of the Ciena transponder Wavelogic 3 .

Figure 2 shows the three examined HPL-FOPA variants. They share most of the components with each other. An optical circulator guided the input signal to a polarization beam splitter (PBS). The PBS splits an input signal into components propagating clockwise $(\mathrm{CW})$ and counter-clockwise $(\mathrm{CCW})$ respectively. An OSA connected via a pair of calibrated $1 \%$ tap couplers measured optical spectra of signal components as they entered and left the loop to monitor to evaluate the HPL-FOPA polarisation dependent gain (PDG). All variants of the loop contained two lengths $(250 \mathrm{~m}$ and $200 \mathrm{~m})$ of HNLF, two pairs of WDM filters, and a polarisation controller (PC). The HNLF parameters were a zero-dispersion wavelength of $\sim 1564 \mathrm{~nm}$, a dispersion slope of $\sim 84 \mathrm{~s} \cdot \mathrm{m}^{-3}$ and a nonlinearity coefficient of $8.2 \mathrm{~W}^{-1} \cdot \mathrm{km}^{-1}$. Each pair of WDMs coupled a pump in a HNLF length and then removed it into a beam dump. Each length of HNLF was therefore a gain medium for a signal component copropagating with a pump. Polarisation of each pump was aligned with polarisation of the corresponding signal using a PC. A PC inside the loop ensured signal components to recombine in the PBS with minimal loss.

Gain - Loss (GL)

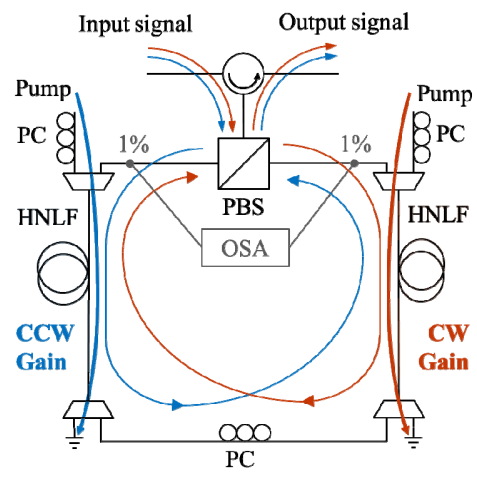

Gain - Filter - Loss (GFL)

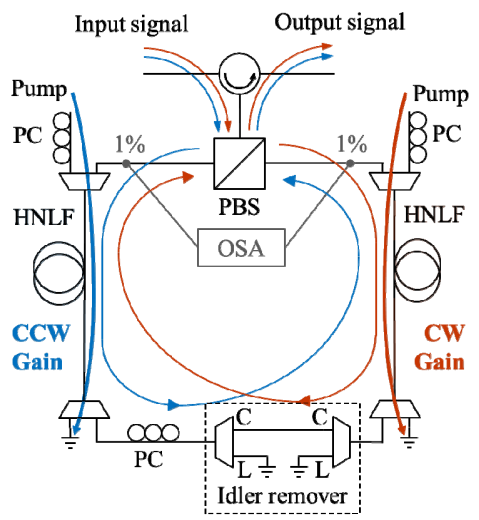

Loss - Gain (LG)

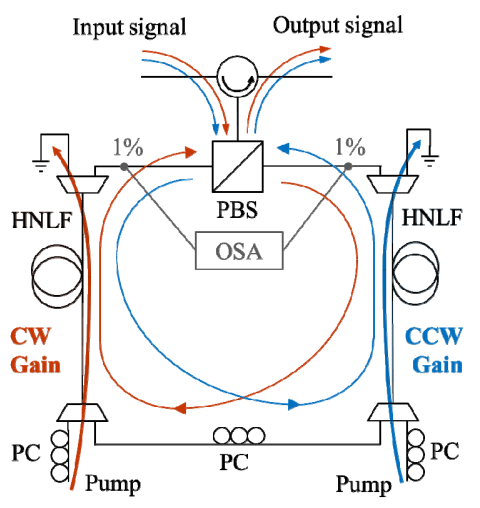

Figure 2. Setups of the examined HPL-FOPA variants.

The difference between the HPL-FOPA variants is the injection point of pumps and a presence of an idler remover in the GFL (Fig. 2). The idler remover consisted of two C/L band splitters connected to allow the signals in the $\mathrm{C}$ band to pass through, while removing idlers generated in the $\mathrm{L}$ band.

A pump seed was sourced from a $100 \mathrm{kHz}$ linewidth laser at $1564.4 \mathrm{~nm}$, phase modulated with a combination of tones with frequencies of 100,320 and $980 \mathrm{MHz}$ and split in two pumps with a $50 \%$ coupler. The two pumps were independently amplified by high power EDFAs with their powers tuned to ensure the $100 \mathrm{G}$ channel net gain of $14 \pm 0.2 \mathrm{~dB}$ and $\mathrm{PDG}<0.2 \mathrm{~dB}$ for all measurements. Pump powers in the $250 \mathrm{~m}$ and the $200 \mathrm{~m}$ lengths of HNLF were in ranges of $1.9 . .2 .4 \mathrm{~W}$ and $2.4 \ldots 2.9 \mathrm{~W}$ respectively.

Although three PCs are present in the HPL-FOPA, the HPL-FOPA is a polarisation-insensitive FOPA due to the following crucial differences from polarisation-sensitive FOPAs. First, the HPL-FOPA is capable of providing the same gain for every signal polarisation and is capable of amplifying polarisation-multiplexed signals. Second, these PCs are used only to compensate for slow drifts within the loop, while the polarisation control of the input signal is not required. Finally, these internal to the loop PCs can be either removed if polarisation-maintaining fibres are employed [10] or fully automated using a feedback from the OSA.

\section{RESULTS AND DISCUSSION}

Figure 3 shows net gain and polarisation dependent gain of every WDM channel at input power of $-25 \mathrm{dBm}$ per channel for all examined HPL-FOPA variants. Net gain of $12.8 \pm 1.5 \mathrm{~dB}$ and PDG $<0.8 \mathrm{~dB}$ are observed across 21 WDM channels spanning over $8 \mathrm{~nm}$. The gain flatness or bandwidth could be improved if WDM channels were moved or extended towards longer wavelengths. The PDG could be improved by optimizing pump wavelengths and/or by employing sections of HNLF with equal length and insertion loss. 


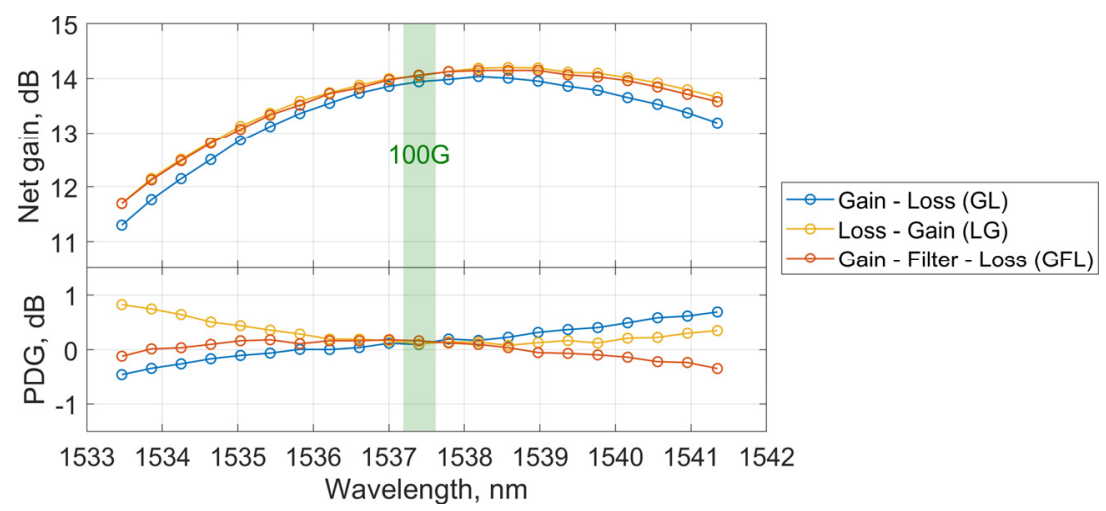

Figure 3. Small signal net gain and polarization-dependent gain (PDG) for all channels and HPL-FOPA variants. A wavelength band of the $100 G$ channel is highlighted.

The HPL-FOPA noise figure $(N F)$ was calculated using Eq. (1) [15], where $N_{\text {output }}$ and $N_{\text {input }}$ are the noise powers measured at the input and the output of the HPL-FOPA with the factual OSA resolution $B$ of $19 \mathrm{GHz}$ at the $100 \mathrm{G}$ channel frequency $v$ of $195 \mathrm{GHz}$, and $G$ is the HPL-FOPA net gain. The $100 \mathrm{G}$ channel was rejected by the WSS in the WDM transmitter for noise power measurement at its frequency. The implemented noise figure measurement technique accounts for an impact of nonlinear crosstalk by including the nonlinear crosstalk power as a part of noise power [16]. The NF measurement error was estimated to be $<1 \mathrm{~dB}$.

$$
N F=\frac{N_{\text {output }}}{G h v B}+\frac{1}{G}-\frac{N_{\text {input }}}{h v B}
$$
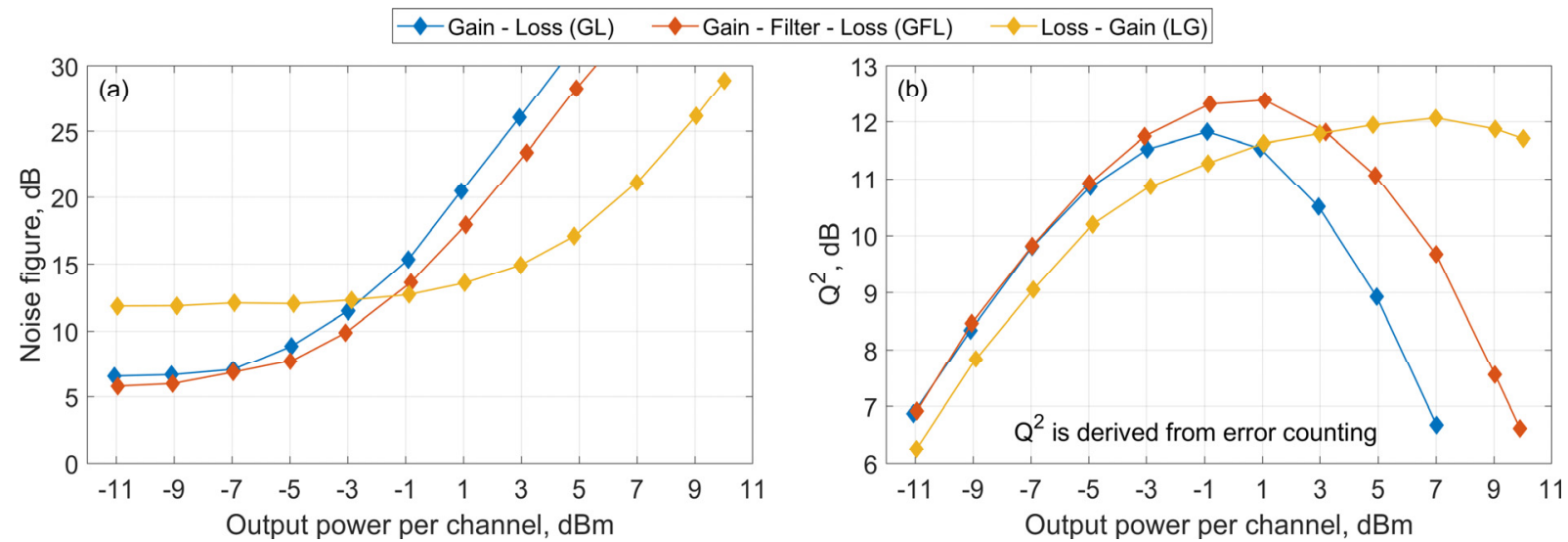

Figure 4. HPL-FOPA characterization as input power per channel is varied in terms of (a) noise figure at the $100 G$ channel frequency and (b) $Q^{2}$ of the $100 G$ channel.

Figure 4(a) shows the NF measured at the 100G channel frequency for each HPL-FOPA variant as input power per channel was varied. A minimum NF of $\sim 6 \pm 1 \mathrm{~dB}$ was measured for GL and GFL variants at low signal power. However, an increase of nonlinear crosstalk power by $3 \mathrm{~dB}$ per $1 \mathrm{~dB}$ of signal power [17] causes a sharp NF degradation. The GFL shows the same NF as the GL at $\sim 1 \mathrm{~dB}$ higher signal power owing to the GFL improved nonlinear crosstalk tolerance. The LG has demonstrated a further $\sim 5 \mathrm{~dB}$ improvement of the $\mathrm{NF}$ tolerance to the nonlinear crosstalk induced degradation. However, the LG minimum noise figure was $6 \mathrm{~dB}$ worse than the minimum noise figure of the GL and the GFL variants thus restricting the viability of the LG.

Figure 4(b) shows $Q^{2}$ of the $100 \mathrm{G}$ channel as input signal power was varied. Equation (2) was used to derive $\mathrm{Q}^{2}$ from BER obtained via counting errors. The GL and the GFL demonstrated the same $\mathrm{Q}^{2}$ when output power per channel was $<-5 \mathrm{dBm}$. As signal power increased the GFL showed up to $3.5 \mathrm{~dB}$ higher $\mathrm{Q}^{2}$ than the GL and had $\sim 1 \mathrm{~dB}$ higher performance peak. This agrees well with the NF measurement results. The LG had $1 \mathrm{~dB}$ lower $\mathrm{Q}^{2}$ than the GL and the GFL at low signal power, but showed superior $\mathrm{Q}^{2}$ for power per channel $>3 \mathrm{dBm}$. This proves that LG is competitive with the GL and the GFL only when signal power is high.

$$
Q^{2}(d B)=20 \times \log _{10}\left[\sqrt{2} \times \operatorname{erf}^{-1}(2 \times B E R)\right]
$$

Overall, the GFL is viewed to be the best performing variant for broadband communications and many other application scenarios as it allows for the EDFA-like noise figure of $\sim 6 \mathrm{~dB}$ whilst being less prone to nonlinear impairments than the GL variant. The GL variant might be the most suitable for realisation of polarisationinsensitive phase-sensitive amplification, because it allows for low noise figure and allows to keep idlers. 
Finally, the LG variant is for applications where the output signal power is more important than the noise figure, e.g. for extended-reach PON requiring to maximise power budget whilst employing only one amplifier [8].

\section{CONCLUSIONS}

We compare three variants of polarisation-insensitive HPL-FOPA whilst amplifying 21 WDM channels within $8 \mathrm{~nm}$ band by $12.8 \pm 1.5 \mathrm{~dB}$. We demonstrate "Gain - Loss" and "Gain - Filter - Loss" variants to provide an EDFA-like noise figure of $6 \mathrm{~dB}$. We suggest the "Gain - Filter - Loss" variant to be employed for broadband communications for its nonlinear crosstalk tolerance. However, the "Gain - Loss" HPL-FOPA is recommended for a polarisation-insensitive phase-sensitive FOPA. The "Loss - Gain" is for applications demanding high output power and with relaxed noise figure requirements, e.g. for reach extension in passive optical networks.

\section{ACKNOWLEDGEMENTS}

The work was funded by EPSRC projects UPON (EP/M005283/1) and FPA-ROCS (EP/R024057/1). The data reported in this paper is available at https://doi.org/10.17036/researchdata.aston.ac.uk.00000467.

\section{REFERENCES}

[1] J. Hansryd, P. A. Andrekson, M. Westlund, J. Li, and P. O. Hedekvist: Fiber-based optical parametric amplifiers and their applications, IEEE J. Sel. Top. Quantum Electron., vol. 8, no. 3, pp. 506-520, 2002.

[2] M. E. Marhic, K. K. Y. Wong, and L. G. Kazovsky: Wide-band tuning of the gain spectra of one-pump fiber optical parametric amplifiers, IEEE J. Sel. Top. Quantum Electron., vol. 10, no. 5, pp. 1133-1141, 2004.

[3] V. Gordienko, M. F. C. Stephens, F. M. Ferreira, and N. J. Doran: Raman-amplified pump and its use for parametric amplification and phase conjugation, Optical Fiber Technol., vol. 56, p. 102183, 2020.

[4] V. Gordienko, M. F. C. Stephens, A. E. El-Taher, and N. J. Doran: Ultra-flat wideband single-pump Raman-enhanced parametric amplification, Optics Express, vol. 25, no. 5, pp. 4810-4818, 2017.

[5] J. M. C. Boggio, S. Moro, E. Myslivets, J. R. Windmiller, N. Alic, and S. Radic: 155-nm continuous-wave two-pump parametric amplification, IEEE Photonics Technol. Lett., vol. 21, no. 10, pp. 612-614, 2009.

[6] Z. Tong, C. Lundström, P. A. Andrekson, C. J. McKinstrie, M. Karlsson, D. J. Blessing, E. Tipsuwannakul, B. J. Puttnam, H. Toda, and L. Grüner-Nielsen: Towards ultrasensitive optical links enabled by low-noise phase-sensitive amplifiers, Nat. Photonics, vol. 5, no. 7, pp. 430-436, 2011.

[7] G.-W. Lu, M. E. Marhic, and T. Miyazaki: Burst-mode amplification of dynamic optical packets using fibre optical parametric amplifier in optical packet networks, Electron. Lett., vol. 46, no. 11, pp. 778-780, 2010.

[8] C. B. Gaur, F. Ferreira, V. Gordienko, A. Iqbal, W. Forysiak, and N. Doran: Comparison of erbium, Raman and parametric optical fiber amplifiers for burst traffic in extended PON, in Proc. Optical Fiber Communication Conference (OFC 2020), paper W4B.3.

[9] M. F. C. Stephens, M. Tan, V. Gordienko, P. Harper, and N. J. Doran: In-line and cascaded DWDM transmission using a $15 \mathrm{~dB}$ net-gain polarization-insensitive fiber optical parametric amplifier, Opt. Express, vol. 25, no. 20, pp. 24312-24325, 2017.

[10] S. Takasaka and R. Sugizaki: Polarization insensitive fiber optical parametric amplifier using a SBS suppressed diversity loop," in Proc. Optical Fiber Communication Conference (OFC 2016), paper M3D.4.

[11] M.F.C. Stephens, V. Gordienko, and N. J. Doran: $20 \mathrm{~dB}$ net-gain polarization-insensitive fiber optical parametric amplifier with >2 THz bandwidth, Opt. Express, vol. 25, no. 9, pp. 10597-10609, 2017.

[12] M. Jazayerifar, I. Sackey, R. Elschner, T. Richter, L. Molle, P. W. Berenguer, C. Schubert, K. Jamshidi, and K. Petermann: Impact of Brillouin backscattering on signal distortions in single-fiber diversity loop based polarization-insensitive FOPAs, J. Light. Technol., vol. 35, no. 19, pp. 4137-4144, 2017.

[13] M. F. C. Stephens, V. Gordienko, and N. J. Doran: Reduced crosstalk, polarization insensitive fiber optical parametric amplifier (PI FOPA) for WDM applications, in Proc. Optical Fiber Communication Conference (OFC 2018), paper W3D.4.

[14] V. Gordienko, F. M. Ferreira, V. Ribeiro, and N. J. Doran: Suppression of nonlinear crosstalk in a polarization insensitive FOPA by mid-stage idler removal, in Proc. Optical Fiber Communication Conference (OFC 2019), paper M4C.4.

[15] D. M. Baney, P. Gallion, and R. S. Tucker: Theory and measurement techniques for the noise figure of optical amplifiers, Optical Fiber Technol., vol. 6, no. 2, pp. 122-154, 2000.

[16] A. D. Szabo, V. Ribeiro, V. Gordienko, F. Ferreira, C. Gaur, and N. J. Doran: Verification of signal-tocrosstalk measurements for WDM fiber optical parametric amplifiers, in Proc. Conference on Lasers and Electro-Optics (CLEO 2020), paper JTu2E.1.

[17] V. Gordienko, M. A. Z. Al-Khateeb, F. M. Ferreira, A. D. Ellis, and N. J. Doran: Unwanted four-wave mixing in fibre optical parametric amplifiers, in Proc. International Conference on Transparent Optical Networks (ICTON 2019), paper Fr.C4.4. 